\title{
Hacia un sistema de gestión ambiental como parte del marco regulatorio de las gasolineras en México
}

On the way to an Environmental Management System as Part of the Regulatory Framework for Gas Stations in Mexico

Por um sistema de gestão ambiental como parte do marco regulatório dos postos de gasolina no México

\author{
Claudia Canobbio-Rojas \\ Universidad Autónoma de Sinaloa. Culiacán, México \\ canobbio@uas.edu.mx \\ https://orcid.org/0000-0003-1670-4320 \\ Berenice Cárdenas-Aragón \\ Universidad Autónoma de Sinaloa. Culiacán, México \\ berenice.cca81@gmail.com \\ https://orcid.org/0000-0002-5672-0707
}

DOI: https://doi.org/10.32719/25506641.2020.8.6

Presentado: 19 de febrero de 2020 - Revisado: 3 de marzo de 2020

Aceptado: 29 de abril de 2020

Artículo de investigación

Licencia Creative Commons

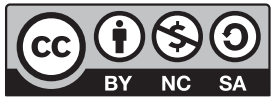




\section{Resumen}

Si bien el cuidado al medioambiente representa oportunidades para las entidades que se rigen bajo una conciencia ecológica, dicha transición hacia una gestión ambiental empresarial también plantea desafíos. Mediante un abordaje metodológico mixto, se proyecta una investigación documental y analítica, así como una encuesta a distintos actores involucrados, con el objetivo de analizar el proceso de configuración de un sistema de gestión ambiental por el cual están atravesando las estaciones de servicio de expendio al público de gasolina y diésel (gasolineras) en México, bajo el nuevo marco normativo de la reforma energética nacional. A través de los hallazgos del presente estudio, se logra dilucidar cómo, en nombre del medioambiente, dichos requerimientos legales se adentran en regular nuevos y diversos aspectos de la gestión de esta clase de firmas, tal como la implementación del llamado Sistema de Administración de Seguridad Industrial, Seguridad Operativa y Protección al Medio Ambiente (SASISOPA). Estas aproximaciones, bajo el enfoque teórico del ciclo de Deming, posibilitan conocer la formulación e instrumentación de proyectos de mejoramiento de la calidad para las estaciones de servicio y su relación funcional con la obtención de asesoría externa.

Palabras clave: Sistema de gestión ambiental, legislación ambiental, medioambiente y comercio; gasolineras.

JEL: Q56 Comercio y medioambiente.

\section{Summary}

Although awareness of the environment represents opportunities for entities that are governed by an ecological conscience, such transition towards corporate environmental management also sets challenges. Through a mixed methodological approach, a documentary and analytical investigation is projected, as well as a survey of different actors involved, with the objective of analysing the process of setting up an environmental management system through which the service stations of public gasoline and diesel (gas stations) in Mexico are, under the new regulatory framework of the national energy reform. Through the findings of this study, it is possible to elucidate how, in the name of the environment, these legal requirements go into regulating new and various aspects of the management of this kind of firms, such as the implementation of the so-called Industrial Security Administration System, Operational Safety and Environmental Protection (SASISOPA). These approaches, under the theoretical approach of the Deming cycle, make it possible to know the formulation and implementation of quality improvement projects for service stations and their functional relationship with obtaining external advice.

Keywords: Environmental management system, environmental legislation, environment and trade; gas stations.

JEL: Q56 Trade and environment. 


\section{Resumo}

Embora o cuidado com o meio ambiente represente oportunidades para as entidades geridas a partir de uma consciência ecológica, tal transição para uma gestão ambiental empresarial também apresenta desafios. Através de uma abordagem metodológica mista, propõe-se uma investigação documental e analítica, assim como uma pesquisa direcionada a distintos agentes envolvidos, com o objetivo de analisar o processo de configuração de um sistema de gestão ambiental pelo qual estão passando as estações de serviço de distribuição ao público de gasolina e diesel (postos de gasolina) no México, de acordo com o novo marco normativo da reforma energética nacional. Através dos resultados do presente estudo, é possível elucidar como, em nome do meio ambiente, tais requerimentos legais passam a regular novos e diversos aspectos da gestão desse tipo de etabelecimento, tal como o faz a implementação do chamado Sistema de Administração de Segurança Industrial, Segurança Operativa e Proteção ao Meio Ambiente (Sistema de Administración de Seguridad Industrial, Seguridad Operativa y Protección al Medio Ambiente-SASISOPA). Tais abordagens, sob o enfoque teórico do ciclo de Deming, possibilitam conhecer a formulação e instrumentação de projetos de melhoria da qualidade voltados para as estações de serviço e sua relação funcional com a obtenção de assessoria externa.

Palavras-chave: Sistema de gestão ambiental, legislação ambiental, meio ambiente e comércio; postos de gasolina.

JEL: Q56 Comércio e meio ambiente.

\section{Introducción}

L

a presente investigación se inserta en la multidisciplinariedad que avoca el análisis de un fenómeno social, cada vez más frecuente, en el que el sujeto de estudio es una entidad económica, regulada por actores públicos, en torno a un contexto medioambiental comprometido.

Temas como el cambio climático, desarrollo sostenible, y demás cuestiones en torno al cuidado del medioambiente, se han insertado de manera más frecuente y profunda en las agendas científicas y políticas, intensificando, a su vez, el desarrollo y la implementación de acuerdos internacionales (Buck 2013); los cuales, a través del derecho internacional ambiental, avanzan en el establecimiento de tratados ambientales multilaterales con acciones, tanto vinculantes como no vinculantes, que plantean líneas de acción y protocolos de tipo softlaw. Sin embargo, los Estados, en función de su soberanía, ejercen la libertad de definir sus políticas ambientales en base a sus intereses políticos y económicos (Morales 2011; Hernández-Huerta, Pérez-Maqueo y Zamora 2018). 
Por su parte, la industria petrolera efectúa un gran número de procesos con repercusiones directas en el medioambiente, vinculadas con la producción, refinamiento, distribución y consumo de petróleo y sus derivados. Para contrarrestarlo, Guédez, De Armas, Reyes y Galván (2003), señalan que entre las principales estrategias empleadas para reducir dicho impacto, se encuentra el sistema de gestión ambiental (SGA), el cual está estrechamente relacionado con el sistema de gestión de calidad, que son mecanismos que proporcionan un proceso sistemático y cíclico de MC.

Al respecto, la adopción del SGA y la obtención de certificaciones se han vuelto una práctica común entre empresas y organismos públicos, los cuales están habituados a la adopción de sistemas de control y gestión de calidad, del tipo de certificación en normas de la Organización Internacional para la Estandarización (ISO) o el Reglamento Comunitario de Ecogestión y Ecoauditoría (EMAS), pensados para contribuir a la gestión de procesos de una organización en torno a sistemas de calidad, medioambiente, responsabilidad social y seguridad.

En el ámbito internacional, diversos estudios han abordado esta problemática (Acuña, Figueroa y Wilches 2017; Granero y Ferrando 2007; González-Torre, Sarkis, Adenso-Díaz 2008; Guédez et al. 2003; Hillary 2004; Lozano y Vallés 2007; Nadel, Galliano y Orozco 2016; Roberts y Robinson 1999), en busca de ventajas y desventajas de los SGA, corroborando la funcionalidad de estas estrategias de gestión como herramientas vitales para la obtención de ventajas competitivas.

En México, como parte de este nuevo esbozo regulatorio, los establecimientos económicos, dedicados al expendio de combustibles, se adentran a un esquema de obligatoriedad, en el cual, destaca el desarrollo e implementación del llamado Sistema de Administración de Seguridad Industrial, Seguridad Operativa y Protección al Medio Ambiente (SASISOPA), diseñado como un modelo de gestión integrado para actividades reguladas del sector hidrocarburos (MX ASEA 2019), reforzando el contexto normativo en materia medioambiental al que cada vez más se ven inmersas todo tipo de empresas, no siendo este giro la excepción (Bakaeva, Garmonov y Sheps 2019).

En lo concerniente, se plantean interrogantes en torno a: ¿cuál es el marco normativo impuesto a esta clase de estaciones de servicio en materia de impacto ambiental?, o ¿cuál es el proceso que siguen estas empresas para 
adaptar sus funciones bajo un esquema de seguridad industrial y operativa desde lo ambiental? Lo anterior, con el propósito de conocer cómo administradores de aquellas unidades económicas dedicadas al expendio al público, de gasolina y diésel en México, perciben y enfrentan el cumplimiento de obligaciones y documentación auditables ante las distintas autoridades. Así pues, se plantea como objetivo de investigación analizar el proceso de configuración de un SGA por el que están atravesando dichas gasolineras, dentro del nuevo marco normativo, tras la reforma energética en este país.

Ahora bien, según el Instituto Nacional de Estadística y Geografía, en México existen 13.920 establecimientos clasificados bajo el código 468411 referido al comercio al por menor de gasolina y diésel, de los cuales se pretende hacer una aproximación del escenario regulatorio al cual están ligados (MX INEGI 2019).

\section{Revisión de literatura}

Para abordar el tema de prevenir o contrarrestar el deterioro ambiental y la sustentabilidad, se parte, por añadidura, del carácter multisectorial y sistémico de los temas ambientales; así como por la no-linealidad de determinados fenómenos productivos, ambientales y sociales. Así, se ha pasado por un largo proceso de identificación de problemas del detrimento ambiental antropogénico; pero finalmente se aceptó que, a mediano y largo plazos, se presentarían situaciones de deterioro que harían peligrar los equilibrios ecológicos y la salud humana, no solo en países industrializados (Wan et al. 2020), sino además en países en vías de desarrollo semiindustrializados (Nadal 2007).

Lo anterior supone una integración de esfuerzos, compromisos y un sentido sinérgico por parte del Estado, comunidad científica, iniciativa privada, organizaciones no gubernamentales y sociedad civil en general, que fundamente su actuar en bases éticas que cambien la idea de subordinación de hombre-naturaleza. Es decir, hay muchos impedimentos y desafíos hacia la implementación de los SGA, debido a la falta de infraestructura, física y reglamentaria, que no pueden resolverse únicamente con solo formulación y ejecución de políticas públicas (Massoud et al. 2010). 
Un referente latinoamericano es el estudio Gestión ambiental en América Latina y el Caribe: evolución, tendencias y principales prácticas, de Manuel Rodríguez y Guillermo Espinoza, publicado en 2012 por el Banco Interamericano de Desarrollo, el cual ha resaltado la necesidad de "una movilización de recursos y de capacidades para fortalecer la gestión ambiental y buscar un mayor cumplimiento de las legislaciones y políticas ambientales, además de crear los mecanismos de control ambiental y de mercado que incidan en las actividades productivas en la región" (Rodríguez y Espinoza 2012, 2). Bajo esta lógica, se presenta la implementación de este tipo de normas en el caso de mejoramiento de combustibles en México, país donde la gestión ambiental, si bien se ejerce en los tres niveles de gobierno, el federal es el que mantiene una mayor centralización dentro de dicha materia (Carrillo y Casellas 2016; Martínez, Santillán y de la Vega 2016; Torres 2014; Merchand 2015; Gutiérrez 2016).

Desde la perspectiva de la ISO, un "sistema de administración ambiental es una herramienta destinada a ser utilizada por una organización que busca gestionar sus responsabilidades ambientales de manera sistemática, que contribuya al pilar ambiental de la sostenibilidad" (ISO 2015, párr. 1). Estos modelos de calidad utilizan indistintamente los términos "administración ambiental" y "gestión ambiental" (Escobar 2009), refiriéndose a las normas que ayudan a una organización a lograr los resultados previstos de su SGA, al proporcionar valor al medioambiente, a la organización misma y a las partes interesadas (Pérez y Bejarano 2008; Boiral, Guillaumie y Heras 2018). Sin embargo, el grado de madurez en materia de gestión ambiental está aún disperso entre las pymes, que van "desde solo cumplir con normativa básica, hasta empresas con sistemas de gestión ambiental y oferta de productos verdes" (González 2017, 62); del mismo modo que, dependiendo de sus recursos y capacidades, estas responden de diferentes formas a las fuerzas institucionales a la hora de desarrollar sus propios SGA (Kang y He 2018; Prakash y Potoski 2006).

Así pues, de acuerdo con la política ambiental de la organización, los resultados previstos de un SGA varían su impacto en términos de: mejora del desempeño ambiental, cumplimiento de obligaciones y logro de objetivos ambientales (Vásquez, Castillo y Mosquera 2018); en otras palabras, se hace referencia a tareas específicas en torno a la búsqueda de la mitigación de 
los impactos negativos como parte de las operaciones, prevenir la polución, manejar la energía de forma eficiente, reducir los residuos, además de la conservación de los recursos (Rivas 2011).

Bajo esta lógica, la literatura señala elementos de entendimiento y aplicación de los SGA, mismos que parten del ciclo Deming-Schewhart, de mejoramiento continuo (planificar, hacer, verificar y actuar), y que, a su vez, permiten identificar las fallas comunes presentes en los procesos de implementación del modelo. Dichos criterios teóricos, pautan variables de análisis, empleadas para este estudio, a partir de su abordaje sobre: política ambiental; planificación; implementación y operación; verificación y acción correctiva; revisión por parte de la gerencia y mejoramiento continuo (Deming 1998; Carro y Carro 2008).

Asimismo, se analiza el ciclo a partir de la guía y medición de etapas de implementación de un sistema de gestión, tales como: “1. Diagnóstico o direccionamiento estratégico; 2 . mapeo de procesos; 3 . política/plan de calidad; 4. procedimientos de trabajo; 5 . capacitación y participación de personal; 6. implementación; 7. auditoría interna; 8. revisión general; 9. acciones correctivas y preventivas; 10. procesos de análisis y mejora; 11. auditoría externa; y 12. certificación del SGA" (Zabihollah y Elam 2000; Fraile 2007; ISO 2019; Hoyos y García 2009, 67). Además, indicadores asociados a la asesoría, auditoría y consultoría administrativa para el fortalecimiento empresarial (Bonilla 2010; Murcia 2016).

En resumen, se habla de todo un marco reglamentario adoptado para responder de forma eficaz a los cambios en las normativas sociales, económicas y competitivas, en conjunto con los aspectos medioambientales. No obstante, quizá el aspecto que mayor influencia tiene en la adopción de este tipo de prácticas ambientales es la presión gubernamental (Delmas y Toffel 2004).

\section{Metodología}

El presente estudio se traza bajo un paradigma mixto en torno al diseño, recolección de datos y tipo de análisis de información. Se recurre al estudio cualitativo a través de la revisión documental y el análisis de contenido, con la búsqueda de unidades de registro relacionadas con códigos preestableci- 
dos sobre: medioambiente, sistema de gestión y estaciones de servicio. Asimismo, se emplea un abordaje cuantitativo con el análisis de los resultados obtenidos por una encuesta, por medio de la regresión logística, perfilada como un procedimiento capaz de predecir el avance en las etapas de implementación de un SGA en las estaciones de servicio de expendio de combustibles, sobre la base de la frecuencia de obtención de asesoría externa en torno a la normativa, los procesos de auditoría y control de calidad que estas unidades económicas solicitan con la finalidad de dar cumplimiento a la nueva reglamentación para el sector hidrocarburos.

Como técnica de recolección de datos se empleó una encuesta, cuyo cuestionario obtuvo validación por juicio de expertos, con una prueba piloto a 15 gasolineras con un alfa de Cronbach de .826 , lo cual señala un grado "bueno" de fiabilidad de consistencia interna del instrumento.

La muestra empleada es no probabilística, partiendo de un padrón de 60 estaciones de servicio, obtenido de una unidad de verificación acreditada (UVA) cuya relación con las gasolineras es la verificación y calibración de bombas expendedoras de combustibles. De los 60 cuestionarios enviados se obtuvo respuesta válida de 51 establecimientos, dado que no se recibió réplica de 7 y 3 no completaron el cuestionario. Los establecimientos encuestados pertenecen a distintos estados del país, como Jalisco, Sinaloa, Sonora, Tamaulipas y Veracruz.

Dentro del abordaje cualitativo, se recurre al análisis de contenido, con una muestra documental concerniente a la revisión del orden constitucional, leyes y normas oficiales mexicanas (NOM) en materia energética, de hidrocarburos y medioambiental, tales como:

i. Reforma energética. DOF20-12-2013.

ii. Ley de hidrocarburos. DOF15-11-2016.

iii. Ley de la Agencia Nacional de Seguridad Industrial y de Protección al Medio ambiente del sector hidrocarburos (ASEA). DOF11-08-2014.

iv. NOM-005-ASEA-2016. DOF7-11-2016.

Dicha aproximación se basó en el análisis exploratorio de datos cualitativos, partiendo de unidades de muestreo, registro y contexto, de los cuales se identificaron códigos y conformaron categorías analíticas, o familias de códigos, planteadas como: medioambiente, sistemas de gestión y estaciones de servicio. 
Se empleó la regresión logística para el análisis de predicción sobre el nivel de avance en la implementación del SGA, partiendo de categorías analíticas, previamente identificadas en los referentes teóricos y determinadas en diversidad de estudios previos (Zabihollah y Elam 2000; Fraile 2007; Carro y Carro 2008; Hoyos y García 2009; Murcia 2016; ISO 2019). Para dicho abordaje se trazaron las variables:

- Nivel de avance SGA: etapa de avance en la implementación del sistema de gestión ambiental por parte de la estación de servicio, bajo el esquema de mejora continua del ciclo de Deming.

- Dependencia de terceros asesores: frecuencia de subcontratación de servicios de despachos de asesoría referente a normativa, procesos de auditoría y control de calidad para la implementación del SGA.

- Grado de involucramiento (alta dirección): tipo de acciones emprendidas y participación activa en procesos de toma de decisiones y retroalimentación durante la configuración del SGA.

Las variables a medir en el instrumento de recolección de datos, se esbozaron en ítems como: ¿se realiza un diagnóstico del estado actual de la gasolinera para proyectar la implementación de un SGA?, ¿la estación cuenta con el apoyo y participación continua de la dirección/gerencia en el mantenimiento del SGA?, ¿se brinda capacitación a los empleados sobre conceptos básicos y se sensibiliza acerca de la implantación del SGA?, ¿con qué frecuencia se recurre a un asesor/consultor externo para cumplir con la normatividad gubernamental y la implementación de un SGA?, ¿con qué finalidad la estación se adentra en el proceso de certificación y aplicación del ciclo de mejora continua?

La identificación, sistematización y descripción de la información recabada precisó el análisis de contenido a través del software Atlas.ti, y la estadística descriptiva e inferencial, con el programa informático SPSS.

\section{Presentación de resultados y análisis}

Por un lado, la técnica de análisis de datos empleada fue el análisis de contenido, y por medio de un análisis exploratorio de datos cualitativos se recurrió a documentos de orden constitucional, leyes y normas oficiales mexi- 
canas en busca de componentes semánticos relacionados con categorías analíticas sobre medioambiente, sistemas de gestión y gasolineras. Asimismo, se empleó la regresión logística con la finalidad de corroborar el efecto de predicción entre el grado de avance en las etapas de implementación de un SGA en las gasolineras, de conocerse la frecuencia de obtención de asesoría externa en torno a la normativa, los procesos de auditoría y control de calidad.

\section{Nuevo entorno regulatorio en materia ambiental}

El abordaje del análisis de contenido precisó la aproximación a las unidades de muestreo y, de modo sintáctico, se realizó la búsqueda de unidades de registro relacionadas con el tema de estudio. El primer documento analizado fue la reforma energética, la cual plantea diversos objetivos en torno a "garantizar estándares internacionales de eficiencia, calidad y confiabilidad de suministro energético" (MX Secretaría de Energía 2015, 3).

Por su parte, la Comisión Reguladora de Energía (CRE), a través de la Ley de Hidrocarburos, Ley de Transición Energética, Ley General de Cambio Climático, entre otras, se faculta como órgano regulador coordinado, con autonomía técnica y de gestión, para el ejercicio de las atribuciones y despacho de los asuntos de estas unidades económica dedicadas a la comercialización de gasolinas y diésel (MX CDDHCU 2016).

En tal sentido, se destaca la ASEA como encargada de supervisar y, en su caso, sancionar a contratistas y asignatarios en materia de protección de las personas, los bienes y el medioambiente. Dentro de su normativa, la Ley de ASEA, art. 5, se atribuye: "Regular a través de lineamientos, directrices, criterios u otras disposiciones administrativas de carácter general necesarias en las materias de su competencia y, en su caso, normas oficiales mexicanas, previa opinión de la Secretaría, en materia de protección al medio ambiente" (MX CDDHCU 2014, 4). Como ejemplo, la NOM-005-ASEA-2016 establece especificaciones de seguridad industrial, operativa y protección al ambiente, en diseño, construcción, operación y mantenimiento de estaciones de servicio para almacenamiento, expendio de diésel y gasolinas (ASEA 2016).

Tras el abordaje de las unidades de muestreo, anteriormente señaladas, el análisis de contenido requirió el empleo de un abordaje temático por medio 
de técnicas de: frecuencias, identificación, clasificación temática y búsqueda de palabras en contexto (Andréu 2002).

Como criterio para la categorización, se tomaron los resultados del análisis de frecuencia de palabras y se realizó el análisis de categorías, a partir del siguiente sistema de codificación de tres familias de códigos:

- Medioambiente: ambiente, medioambiental, ecológico, verde, energías limpias, otras.

- Sistema de gestión: administración, sistema de gestión, calidad, mejora continua, otras.

- Unidades de servicio (gasolineras): empresa, hidrocarburos, regulados/ estaciones de servicio, otras.

Tabla 1

Coocurrencias entre categorías de análisis: medioambiente y sistema de gestión

\begin{tabular}{|l|c|c|c|c|c|}
\hline & $\begin{array}{c}\text { Ley de } \\
\text { ASEA }\end{array}$ & $\begin{array}{c}\text { Ley de } \\
\text { Hidrocarburos }\end{array}$ & NOM-005ASEA & $\begin{array}{c}\text { Reforma } \\
\text { energética }\end{array}$ & Total \\
\hline Medioambiente & 14 & 3 & 22 & 5 & 44 \\
\hline Sistema de gestión & 23 & 11 & 1 & 2 & 37 \\
\hline Totales & 37 & 14 & 23 & 7 & 81 \\
\hline
\end{tabular}

Fuente y elaboración propia.

Del marco regulatorio analizado, tal como se muestra en la tabla 1 , se efectuó el análisis de coocurrencias para observar la relación entre categorías, con un total de 81 coocurrencias entre ambas categorías de análisis, todo ello dentro del contexto de códigos establecidos acerca del sector hidrocarburos y gasolineras.

En base a lo anterior, se vislumbra cognición de los riesgos y peligros del quebranto ambiental que surgen de agentes generadores del deterioro; 
entre ellos, las unidades económicas encargadas de la operación, distribución y venta al público de petrolíferos en México, lo cual se hace más evidente dentro de la Ley de ASEA y NOM-005ASEA.

\section{Escenario de imposiciones legales y cumplimientos normativos en materia de medioambiente a gasolineras en México}

El proceso de incursión en un SGA ha generado, en varios actores dentro del sector empresarial, una gran disertación sobre su alcance, pero sobre todo acerca de los retos y dificultades en torno a su adopción. De acuerdo con los datos arrojados por el análisis estadístico descriptivo, la mayoría de los actores empresariales abordados solo reconocen a la CRE y ASEA, con un señalamiento del 95,2 y $100 \%$, respectivamente, como las dependencias a las que deben rendir cuentas del quehacer de su organización relacionado con su impacto ambiental. Sin embargo, omiten otras instituciones como: Secretaría del Trabajo y Previsión Social (STPS), Procuraduría Federal de Protección al Ambiente (PROFEPA) y Procuraduría Federal del Consumidor, por mencionar algunas.

En tal caso, las gasolineras están obligadas a responder ante una serie de requerimientos de regulación ambiental, como: presentación de manifestación de impacto ambiental; registro como generadores de residuos peligrosos; cumplimiento de la Ley General del Equilibrio Ecológico y la Protección al Ambiente; poseción de licencia ambiental única; presentación de un análisis de riesgos; elaboración de un programa interno de protección civil; atención a las NOM de PROFEPA, además de reglamentaciones de la STPS.

Por otro lado, del total de estaciones encuestadas solo un $29,4 \%$ conoce a cabalidad las obligaciones y documentación auditables ante instancias acreditadas; de ahí que, un número considerable de estaciones afirman recurrir a la contratación de servicios de asesores/consultores externos para cumplir con obligaciones y documentación en materia medioambiental (tabla 2). 
Tabla 2

Obligaciones en materia ambiental ante CRE y ASEA proyectadas a 2020

\begin{tabular}{|c|c|c|c|}
\hline Instituciones & Obligaciones & Descripción & $\begin{array}{c}\text { Requiere apoyo } \\
\text { de } \\
\text { especialista }\end{array}$ \\
\hline \multirow[b]{3}{*}{ CRE } & Registro & $\begin{array}{l}\text { - Toda estación de servicio } \\
\text { (obligatorio). } \\
\text { - Única ocasión. }\end{array}$ & \\
\hline & Supervisión anual & $\begin{array}{l}\text { - Fecha límite: } 31 / 01 . \\
\text { - Anual. }\end{array}$ & \\
\hline & $\begin{array}{l}\text { Pruebas de laboratorio } \\
\text { NOM-016-CRE-2016 }\end{array}$ & $\begin{array}{l}\text { - Calidad del producto } \\
\text { (revisión p/tanque). } \\
\text { - Contratación de laboratorio } \\
\text { acreditado por la CRE y testi- } \\
\text { ficación por UVA/tercero espe- } \\
\text { cialista. } \\
\text { - Semestral. }\end{array}$ & $\sqrt{ }$ \\
\hline \multirow{4}{*}{ ASEA } & $\begin{array}{c}\text { Aprovechamientos } \\
\text { de análisis/evaluación } \\
\text { de informes preventivos } \\
\text { de impacto ambiental }\end{array}$ & - Única ocasión. & $\sqrt{ }$ \\
\hline & $\begin{array}{c}\text { Licencia Ambiental } \\
\text { Única }\end{array}$ & - Única ocasión. & $\sqrt{ }$ \\
\hline & $\begin{array}{c}\text { Revisiones } \\
\text { NOM-005-ASEA-2016 }\end{array}$ & - Semestral. & $\sqrt{ }$ \\
\hline & SASISOPA & $\begin{array}{l}\text { - Registro. } \\
\text { - Elaboración del documento. } \\
\text { - Autorización. } \\
\text { - Aprovechamiento. } \\
\text { - Auditoría Anual Interna. } \\
\text { - Bienal. }\end{array}$ & $\sqrt{ }$ \\
\hline
\end{tabular}

Fuente: adaptado de MX CRE (2019); MX ASEA (2016). 
A este respecto, los encuestados señalan una alta frecuencia, 36 "siempre" y 15 "algunas veces", refiriéndose a la solicitud de ayuda de terceros acreditados, UVA, asesores y consultores expertos que realizan verificaciones, a petición de parte, de los procesos de manejo dentro de sus instalaciones y distribución de sus productos. En tanto, el 100\% señalaron que, para las revisiones y dictamen de la NOM-005-ASEA-2016, requieren de un tercero especialista, así como la guía de un despacho experto en la materia, para atender las no conformidades (de existirlas) recibidas por la autoridad, de la revisión documental, física y ocular.

En tal sentido, desde la visión de las unidades económicas en cuestión, la adopción del SASISOPA implica la postulación de un nuevo esquema, con nuevos y diversos rubros de regulación; dado que abarca medidas de reducción de riesgos, esfuerzos y duplicidades, cumplimiento legal, protección de trabajadores, responsabilidad, competitividad y continuidad del negocio (MX ASEA 2019).

Bajo esta lógica, se plantea la regresión logística perfilada como una técnica de análisis capaz de predecir el avance en las etapas de implementación de un SGA en las gasolineras de expendio de combustibles, de conocerse la frecuencia de obtención de asesoría externa en torno a la normativa, los procesos de auditoría y control de calidad, que estas empresas solicitan para poder dar cumplimiento a la nueva reglamentación para el sector hidrocarburos.

Para la predicción del nivel de avance en la implementación del SGA en las estaciones de servicio, en este caso variable dependiente (VD), se categorizó en nivel "bajo" con 11 menciones y "alto" con 40. Mientras que la variable independiente (VI), correspondiente al grado de dependencia de asesoría externa, dio 36 respuestas para "siempre", 15 "algunas veces" y 0 "nunca" (tabla 3).

Como parte del análisis de regresión logística, se muestra el llamado "bloque 0 o inicial", donde el modelo de base se fundamenta en la categoría con mayor frecuencia, lo cual significa que, para la predicción de la variable categórica, conocida como grado de avance en la implementación del SGA, se asume que todos los casos están en un nivel alto; es decir, dentro de las etapas: auditoría interna, revisión general, acciones correctivas y preventivas, procesos de análisis y mejora, auditoría externa; y registro y autorización. Para este análisis de regresión logística, el bloque 0 arrojó un 78,4\% de probabilidad de acierto en el resultado de la VD. 
Tabla 3

Cruce de frecuencia de obtención de asesoría y grado de avance del SGA

\begin{tabular}{|l|c|c|c|}
\hline \multirow{2}{*}{$\begin{array}{c}\text { Frecuencia } \\
\text { obtención de asesoría externa }\end{array}$} & \multicolumn{2}{|c|}{ Grado de avance en implementación de SGA } & \multirow{2}{*}{ Total } \\
\cline { 2 - 3 } & Bajo & Alto & \\
\hline Nunca & 0 & 0 & 0 \\
\hline Algunas veces & 10 & 5 & 15 \\
\hline Siempre & 1 & 35 & 36 \\
\hline Total & 11 & 40 & 51 \\
\hline
\end{tabular}

Fuente y elaboración propia.

La variable incluida en la formulación del modelo arroja una significancia (Sig.) menor de .000, indicando que dicha variable mejora significativamente la predicción de la VD. En este caso, la variable independiente por incluir es la frecuencia de obtención de asesoría externa, referida a la normatividad y procedimientos para la implementación de un SGA por parte de las gasolineras.

El bloque 1 de regresión logística permite dar cuenta de aceptación o rechazo de la hipótesis de investigación. Según resultados, la puntuación de eficiencia estadística de ROA indica que hay una mejora significativa en la predicción de la probabilidad de ocurrencia de las categorías de la VD (Chi cuadrado: 24.948; un grado de libertad (gl):1 y p<.001).

Se obtuvieron puntuaciones de R cuadrado de Cox y Snell con .387 y de Nagelkerke con .597, arrojando un resultado equivalente a $\mathrm{r}$ cuadrada para variable categórica. Ambos resultados son válidos; sin embargo, se elige aquel que exprese la mayor cantidad de varianza explicada de la VD. En este caso, el valor de R cuadrado de Nagelkerke indica que el modelo propuesto explica el 59,7\% de la varianza de la VD.

La tabla 4 muestra las contingencias de resultados observados y pronosticados por el modelo del bloque 1 , señalando un $88,2 \%$ de probabilidad de acierto en el resultado de la VD, al conocerse la frecuencia de subcontratación de un despacho de consultoría. 
Tabla 4

Resultados observados / pronosticados por el modelo

\begin{tabular}{|c|c|c|c|c|c|}
\hline \multirow{6}{*}{ Paso 1} & \multirow{3}{*}{\multicolumn{2}{|c|}{ Observado }} & \multicolumn{3}{|c|}{ Pronosticado } \\
\hline & & & \multicolumn{2}{|c|}{$\begin{array}{l}\text { Grado de avance (implementación } \\
\text { SGA) }\end{array}$} & \multirow{3}{*}{\begin{tabular}{|c|}
$\begin{array}{c}\text { Porcentaje } \\
\text { correcto }\end{array}$ \\
90,9 \\
\end{tabular}} \\
\hline & & & Bajo & Alto & \\
\hline & \multirow{2}{*}{$\begin{array}{l}\text { Grado de avance } \\
\text { (implementación } \\
\text { SGA) }\end{array}$} & Bajo & 10 & 1 & \\
\hline & & Alto & 5 & 35 & 87,5 \\
\hline & \multicolumn{2}{|c|}{ Porcentaje global } & & & 88,2 \\
\hline
\end{tabular}

Fuente y elaboración propia.

En la tabla 5 se señala que $\operatorname{Exp}(B)<1$, cuya interpretación es: si la frecuencia de subcontratación de un asesor externo es igual a 1, el grado de avance es 0 . O bien, cuando la frecuencia de subcontratación de un asesor externo o despacho de consultoría en tema de gestión de calidad e hidrocarburos es de "algunas veces", es más probable que el grado de avance en la implementación de un SGA sea bajo.

Tabla 5

Variables en la ecuación

\begin{tabular}{|c|c|c|c|c|c|c|c|}
\hline \multicolumn{2}{|c|}{} & B & $\begin{array}{c}\text { Error } \\
\text { estándar }\end{array}$ & Wald & gl & Sig. & Exp (B) \\
\hline \multirow{2}{*}{ Paso 1} & $\begin{array}{l}\text { Frecuencia subcontratación } \\
\text { de asesor/consultor externo }\end{array}$ & -4.248 & .153 & 13.586 & 1 & .000 & .014 \\
\cline { 2 - 9 } & Constante & 3.555 & 1.014 & 12.289 & 1 & .000 & 35.000 \\
\hline
\end{tabular}

Fuente y elaboración propia.

La puntuación del Wald para el modelo probado indica que la variable independiente aporta significativamente a la predicción de la VD. Los resultados obtenidos de este modelo se pueden generalizar a la población (Wald:13.586; gl:1; $\mathrm{p}<.001$ ). 
En resumen, se recabaron las tres pruebas necesarias para probar la hipótesis. Por una parte, se sabe que la VI introducida al modelo mejora significativamente la predicción; el modelo que se está probando explica el 78,4\% de la varianza de la VD y, por último, se constata que, al hacer predicciones basadas en el modelo creado, se tiene un $88,2 \%$ de acierto.

Se comprueba, entonces, la hipótesis alternativa Ha, la cual se resume en que la probabilidad de avance en las etapas de implementación de un SGA en las gasolineras de expendio de combustibles se puede predecir por la frecuencia de subcontratación de terceros asesores en torno a la normativa, procesos de auditoría y control de calidad.

\section{Discusión y conclusiones}

A partir del análisis realizado se infiere sobre la reconfiguración regulatoria que ha venido fortaleciendo a las instituciones del Estado en temas medioambientales. Respecto al sector en estudio, el análisis de contenido de documentos oficiales sobre energía e hidrocarburos perfila una reducción en la exposición del país a los riesgos ambientales en las actividades de expendio al público de gasolinas. A través de este ejercicio de investigación exploratoria y descriptiva, el análisis de contenido permitió analizar la normatividad que vincula el quehacer de alrededor de 14.000 gasolineras en México, desde una perspectiva medioambiental; lo cual se deduce del análisis de coocurrencias entre categorías planteadas y en cuyas unidades de contexto se identifican otros beneficios que la nueva regulación prevé en la adopción del SGA, enlistando: la estandarización de procesos, optimización de recursos, satisfacción del cliente, mejora continua, entre otros.

Por otra parte, la transición hacia el sistema de gestión de las gasolineras se ha configurado de manera integral, al buscar no solo las mejoras en el desempeño ambiental, sino además, la seguridad industrial y operativa, convocando a aquellos inmersos en dichas actividades para que conformen su propio sistema de administración, dando cumplimiento de los lineamientos para la conformación, implementación y autorización del SASISOPA, en cuyos elementos están la identificación de peligros y aspectos ambientales 
de las actividades, productos y servicios; además del análisis de riesgo y evaluación de aspectos ambientales.

Bajo esta lógica, la presente investigación obtiene evidencia de que, si bien se justifica la normatividad de impacto ambiental al giro empresarial, en el caso de las gasolineras en México se vislumbra una regulación creciente, compleja y cada vez más profunda, escenario para el cual no siempre están preparadas. Antes de la reforma energética, la normatividad en cuestión se limitaba a escasos requerimientos, impuestos por la empresa estatal Petróleos Mexicanos (Pemex), del tipo de solicitud de resolutivos de impacto al ambiente emitido por alguna entidad federativa.

En específico, se detecta un nivel avanzado en el proceso de implementación del SGA dentro de las gasolineras. Estas, tras haber rebasado las etapas de diagnóstico, mapeo de procesos, establecimiento de una política y plan de calidad, empleo de procedimientos de trabajo, la capacitación, formación y sensibilización del personal (en todos los niveles), así como la implementación, a la fecha proyectan las fases de auditoría interna, revisión general, acciones correctivas y preventivas, procesos de análisis y mejora, auditoría externa y certificación del SGA, o bien, registro y autorización, en el caso de las estaciones de servicios. Al completarse las etapas de implementación se aplica el ciclo de Deming de mejora continua: planificar, hacer, verificar y actuar.

En síntesis, el objetivo de investigación se logra al revelar una serie de hallazgos sobre la forma en que las gasolineras afrontan el cumplimiento de obligaciones en materia medioambiental, a partir de indicios sobre el grado de conocimiento que estas poseen sobre las reglamentaciones y documentación auditables; así como, la necesidad de asesoría/consultoría externa para atender las regulaciones impuestas por las distintas autoridades; y que, de acuerdo con el análisis de regresión logística, la dependencia de despachos de asesoría es una variable de peso en la predicción de dicho avance en la ejecución de un SGA.

Igualmente, se destaca que más allá de reducir las cargas ambientales, a través del seguimiento de la normatividad, este tipo de unidades económicas, priorizan la llamada viabilidad económica, refiriéndose a la carga monetaria que representa adentrarse a dicho cumplimiento legal de impacto ambiental, al desplegarse una serie de imposiciones económicas, además de la obliga- 
toriedad de contar con pólizas de seguros - de responsabilidad civil y daño ambiental-.

Cabe resaltar que este trinomio gobierno-economía-medioambiente se encuentra en un proceso abierto, que proyecta culminar al final del año 2020. A saber, se programan todavía una serie de actividades relacionadas con la obtención de licencias de funcionamiento, certificación del Sistema de Gestión de Medición 2020, dictamen de la NOM-016-CRE-2016, implementación del SASISOPA, además del análisis de calidad de petrolíferos.

Se advierte entonces que el resultado del estudio permite comprender las características de distintas etapas en la configuración de este nuevo esquema de trabajo, lo cual procura ser de utilidad para determinar proyecciones de tiempo en formulación e implementación de un nuevo SGA, además de mejorar la capacidad de asimilación, adopción y retroalimentación del nuevo marco normativo al que están sujetas las gasolineras.

Sobre la base de los hallazgos aquí presentados, se destaca aún más la importancia del rol que desempeñan las comunidades científicas al ocuparse de los temas ambientales. De igual modo, concierne a las autoridades y a los sectores empresariales, así como a la sociedad civil, apoyar las investigaciones que conduzcan a una estrategia integral y efectiva, diseñada a partir de un análisis multidisciplinario, destinado a crear medios de inducción y cumplimiento colectivo de las distintas reglamentaciones en materia medioambiental.

\section{Referencias}

Acuña, Norbert, Lindsay Figueroa y María Wilches. 2017. "Influencia de los Sistemas de Gestión Ambiental ISO 14001 en las organizaciones: caso estudio empresas manufactureras de Barranquilla”. Ingeniare 25 (1) 143-153. 10.4067/S0718-33052017000100143.

Andréu, Jaime. 2002. Las técnicas de análisis de contenido: una revisión actualizada. Sevilla: Fundación Centro de Estudios Andaluces. https://bit.ly/2zKEnzP.

Bakaeva, Natalya, Kirill Garmonov y Roman Sheps. 2019. "Measures to Improve Environmental Safety of Urban Gas Stations". IOP Conference Series: Materials Science and Engineering 687 (6): 1-6. 10.1088/1757-899X/687/6/066015. 
Boiral, Olivier, Laurence Guillaumie e Iñaki Heras. 2018. "Adoption and Outcomes of ISO 14001: A Systematic Review”. International Journal of Management Reviews 20 (2): 411432. 10.1111/ijmr.12139.

Bonilla, Josué. 2010. "La calidad de los sistemas de gestión de recursos humanos en función de la tenencia o no de la certificación ISO 9001:2000, algunas evidencias/indicadores asociados". Estudios Gerenciales 26 (115): 39-64. 10.1016/S0123-5923(10)70111-X.

Buck, Susan. 2013. Understanding Environmental Administration and Law. Washington D. C.: Island Press.

Carrillo, Paola, y Antònia Casellas. 2016. "Descentralización y gestión ambiental en América Latina: un análisis de las publicaciones académicas". Cuadernos de Desarrollo Rural 13 (78): 67-96. 10.11144/Javeriana.cdr13-78.dgal.

Carro, Juan Carlos, y Juan Carro. 2008. "La inteligencia empresarial y el sistema de gestión de calidad ISO 9001: 2000". Ciencias de la Información 39 (1): 31-44. https://bit.ly/2XsC VuX.

Delmas, Magaly, y Michael Toffel. 2004. "Stakeholders and Environmental Management Practices: An Institutional Framework". Business Strategy and the Environment 13 (4): 209-222. 10.1002/bse.409.

Deming, William. 1989. Calidad, productividad y competitividad: la salida de la crisis. Madrid: Díaz de Santos. https://bit.ly/3eHUPiR.

Escobar, Sandra. 2009. "Realidad de los sistemas de gestión ambiental". Sotavento MBA (13): 68-79. https://bit.ly/2SK2jIN.

Fraile, Ana. 2007. "Guía administrativa para implementar el sistema de gestión de calidad en las PYMEs en Boyacá". Semestre Económico 10 (19): 101-112. https://bit.ly/2U3UW0q.

González, Andreína. 2017. "La gestión ambiental en la competitividad de las pymes". Revista Cientifica Agroecosistemas 5 (1): 60-70. https://bit.ly/37Q3LyP.

González-Torre, Pilar, Joseph Sarkis y Belarmino Adenso-Díaz. 2008. "Environmental Management System Certification and Its Influence On Corporate Practices: Evidence From The Automotive Industry". International Journal of Operations \& Production Management 28 (11): 1021-1041. 10.1108/01443570810910179.

Granero, Javier, y Miguel Ferrando. 2007. Cómo implantar un sistema de gestión ambiental según la norma ISO 14001:2004. Madrid: FC Editorial.

Guédez, Carolina, Desirée de Armas, Rosa Reyes y Luis Galván. 2003. "Los sistemas de gestión ambiental en la industria petrolera internacional". Interciencia 28 (9): 528-557. https://bit.ly/37p6Rd7.

Gutiérrez, Raquel. 2016. “¿Está preparado México para el fracking? Reforma energética en México 2014". Sociedad y Ambiente 1 (9): 102-120. 10.31840/sya.v0i9.1635.

Hernández-Huerta, Arturo, Octavio Pérez-Maqueo y Miguel Zamora. 2018. “¿Puede el desarrollo ser sostenible, integral y coherente?". Regions and Cohesion 8 (3): 1-14. $10.3167 /$ reco.2018.080302. 
Hacia un sistema de gestión ambiental como parte del marco regulatorio de las gasolineras en México

Hillary, Ruth. 2004. "Environmental Management Systems and the Smaller Enterprise". Journal of Cleaner Production 12 (6): 561-569. 10.1016/j.jclepro.2003.08.006.

Hoyos, William, y Bertha García. 2009. "Estructura metodológica para gestionar de manera integrada la medición, evaluación y seguimiento de los sistemas de gestión de calidad, medio ambiente y salud ocupacional". Revista Universidad EAFIT 45 (155): 60-73. https:// bit.ly/3gPwauz.

Kang, Yuanfei, y Xinming He. 2018. "Institutional Forces and Environmental Management Strategy: Moderating Effects of Environmental Orientation and Innovation Capability”. Management and Organization Review 14 (3): 577-605. 10.1017/mor.2017.56.

Lozano, Macarena, y José Vallés. 2007. “An Analysis of the Implementation of an Enviromental Management System in a Local Public Administration". Journal of Environmental Management 82 (4): 495-511. 10.1016/j.jenvman.2006.01.013.

Martínez, Francisco, Mónica Santillán y Ángel de la Vega. 2016. "La reforma energética de 2013/2014 y el desarrollo industrial en México: contenidos, implicaciones y propuestas”. Análisis Económico XXXI (78): 7-32. 10.36105/iut.2018n28.02.

Massoud, May, Rabih Fayad, Rabih Kamleh y Mutasem El-Fadel. 2010. "Environmental Management System (ISO 14001) Certification in Developing Countries: Challenges and Implementation Strategies”. Environmental Science and Technology 44 (6): 1884-1887. $10.1021 /$ es902714u.

Merchand, Marco. 2015. "Estado y reforma energética en México". Problemas del desarrollo 46 (183): 117-139. 10.1016/j.rpd.2015.10.006.

Morales, Paola. 2011. "La cooperación internacional para el desarrollo como mecanismo de gestión ambiental: aproximación teórica a la estructura de gestión ambiental global”. Gestión y Ambiente 14 (1): 129-142. https://bit.ly/32gApbM.

Murcia, Héctor. 2016. Auditoría administrativa con base en innovación organizacional. Bogotá: Ediciones de la U.

MX Agencia de Seguridad, Energía y Ambiente (ASEA). 2016. NOM-005-ASEA-2016. Ciudad de México: ASEA. Accedido 18 de agosto de 2019. https://bit.ly/3bDEbjZ.

---. 2019. Sistema de Administración de Seguridad Industrial, Seguridad Operativa y Protección del Medio Ambiente (SASISOPA). Ciudad de México. Accedido 8 de agosto. https:// bit.ly/2UN4nCF.

MX CDDHCU. 2014. Ley de la Agencia Nacional de Seguridad Industrial y de Protección al Medio ambiente del Sector Hidrocarburos. Diario Oficial de la Federación, Ciudad de México, 11 de agosto. Accedido 1 de septiembre de 2019. https://bit.ly/37W9Ee3.

---. 2016. Ley de Hidrocarburos. Diario Oficial de la Federación, Ciudad de México, 15 de noviembre. Accedido 3 de septiembre de 2019. https://bit.ly/2SV7yGR.

MX Comisión Reguladora de Energía (CRE). 2019. "Actividades reguladas en materia de petrolíferos, petroquímicos y bioenergéticos". Ciudad de México: CRE. Accedido 1 de octubre. https://bit.ly/2vx1knC. 
MX INEGI. 2019. "Directorio Estadístico Nacional de Unidades Económicas". Ciudad de México: INEGI. Accedido 15 de agosto. https://bit.ly/2OUgdXN.

MX Secretaría de Energía. 2015. Reforma Energética. Resumen ejecutivo. Ciudad de México. Accedido 20 de septiembre de 2019. https://bit.ly/39eZaYw.

Nadal, Alejandro. 2007. "El desarrollo sustentable: un concepto multidisciplinario en un mundo complejo y cambiante". En Desarrollo sustentable y cambio global, editado por Alejandro Nadal, 93-102. Ciudad de México: El Colegio de México. doi:10.2307/j.ctvhn09kv.

Nadel, Simon, Danielle Galliano y Luis Orozco. 2016. "Adoption of Environmental Management Systems and Organizational Changes: The Case of The French Industrial Firms". Journal of Innovation Economics \& Management 21 (3): 109-132. 10.3917/jie.021.0109.

Organización Internacional de Estandarización (ISO). 2015. Norma de sistema de gestión de calidad-14001:2015. Accedido 15 de enero de 2020. https://bit.ly/37txJbM.

---. 2019. "Etapas fundamentales al implantar un sistema de gestión de calidad". Accedido 21 de noviembre. https://bit.ly/2Xq3HUA.

Pérez, Rafael, y Alexander Bejarano. 2008. "Sistema de gestión ambiental: Serie ISO 14000". Revista Escuela de Administración de Negocios (62): 89-105. https://bit.ly/2UQRWpj.

Prakash, Aseem, y Matthew Potoski. 2006. "Racing to the Bottom Trade, Environmental Governance, and ISO 14001". American Journal of Political Science 50 (2): 350-364. 10.1111/j.1540-5907.2006.00188.x.

Rivas, María. 2011. "Modelo de sistema de gestión ambiental”. Gestión y medio ambiente 14 (1): 151-162. https://bit.ly/325HhJf.

Roberts, Hewitts, y Gary Robinson. 1999. ISO 14001 EMS. Manual de sistemas de gestión medioambiental. Madrid: Paraninfo.

Rodríguez, Manuel, y Guillermo Espinoza. 2012. Gestión ambiental en América Latina y el Caribe: evolución, tendencias y principales prácticas. Banco Interamericano de Desarrollo / Departamento de Desarrollo Sostenible División de Medio Ambiente. https://bit. ly/2Sr6dHS.

Torres, Ramón. 2014. "La reforma energética ¿coadyuva al desarrollo?”. Economía UNAM 11 (32): 120-124. 10.1016/s1665-952x(14)70455-4.

Vásquez, Óscar, María Castillo y William Mosquera. 2018. "Exploratory Review of The State of the Art on the Impact of Implementation in SMEs: Case Study in the Environmental Management System". Conferencia presentada en Proceedings of the International Conference on Industrial Engineering and Operations Management. Washington D. C., 27-29 de septiembre. https://bit.ly/38WDD6X.

Wan, Xue, Xiaoning Yang, Quaner Wen, Jun Gang y Lu Gan. 2020. "Sustainable Development of Industry-Environmental System Based on Resilience Perspective". International Journal of Environmental Research and Public Health 17 (2): 645. 10.3390/ijerph17020645.

Zabihollah, Rezaee, y Rick Elam. 2000. "Emerging 2000. ISO 14000 Environmental Standards: a Step-By-Step Implementation Guide". Managerial Auditing Journal 15 (1/2): 60-67. https://bit.ly/2XrXAza. 